\title{
Sequence effects by non-predictive arrow cues
}

\author{
Qian Qian · Keizo Shinomori • Miao Song
}

Received: 19 January 2011/ Accepted: 27 April 2011/Published online: 12 May 2011

(C) The Author(s) 2011. This article is published with open access at Springerlink.com

\begin{abstract}
Previous studies have found that attention orienting is influenced by the orienting processes of previous trials in a spatial cueing paradigm. This study mainly investigated whether this sequence effect could happen for a non-predictive arrow cue and whether it was influenced by the cue-target SOAs in previous and current trials. A significant sequence effect was observed for arrow cues even when voluntary control was not required, and it was significantly influenced by the SOAs of previous trials. The present results support the automatic memory check hypothesis and may reflect some temporal characteristics of the memory mechanism in sequential processes. In addition, contrary to the previous findings, we found an overall response facilitation following a catch trial, suggesting that the influence of preceding catch trials may be sensitive to experimental contexts.
\end{abstract}

At any one moment, people can attend only to a small part of the world for their limited processing resources. Therefore, it is highly beneficial for the human cognitive system to be able to select pertinent input for further processing. Orienting of attention refers to the alignment of some internal mechanisms with an external sensory input source, which makes people preferentially process that input. Such

Q. Qian $(\bowtie) \cdot$ K. Shinomori · M. Song

Department of Engineering, Graduate School of Engineering,

Kochi University of Technology, Tosayamada-town,

Kami-city, Kochi 782-8502, Japan

e-mail: qianqian1025@gmail.com

K. Shinomori

e-mail: shinomori.keizo@kochi-tech.ac.jp

M. Song

e-mail: songmiaolm@gmail.com ability enables us to detect and respond quickly to potential danger or relevant events. A great deal of research has investigated the orienting to visual input by using the spatial cueing paradigm (e.g., Posner, 1980; Posner \& Cohen, 1984). In a typical example of this paradigm, participants are instructed to respond to the onset of a target that can appear to the left or right of the fixation point by making a rapid key-press response. Before the onset of the target, a cue that indicates one of the possible target locations is presented for a certain time period (i.e., cue-target stimulusonset asynchrony (SOA)). Faster reaction times (RTs) and/ or more accurate performance with targets appearing in the cued location (compared with those in the uncued location) indicate attention shift to the cued location.

Orienting of attention may be elicited and controlled in different ways, and one way to distinguish between different forms of orienting is to examine the effects of different types of attentional cues. Traditionally, there are two major types: exogenous cues, such as sudden onset of peripheral events; and endogenous cues, such as centrallypresented symbolic cues. It was commonly assumed that an exogenous cue automatically attracts attention, because the orienting by it occurs rapidly even though it is not predictive of the actual target location. Furthermore, the cueing effect is not disrupted even if the participants know that the target is more likely to appear in the uncued location (Jonides, 1981; Remington, Johnston \& Yantis, 1992). In contrast, orienting in response to endogenous cues (e.g., a pointing arrow or a directional word, like 'LEFT') appears to be under voluntary control. That is, such cues only shift attention when they correctly predict the target location on most trials to provide an explicit strategy for the participant to orient in the direction of the cue (Posner, Snyder \& Davidson, 1980; Jonides, 1981). Another difference between exogenous and endogenous 
cues is that the RT facilitation effect of exogenous cues emerge rapidly at short SOAs and then change into an inhibition effect at longer SOAs (i.e., slowed RTs at cued location relative to uncued location, inhibition of return (IOR), Maylor, 1985, Posner \& Cohen, 1984), while the RT facilitation effect of endogenous cues sets up slowly and remains stable for long SOAs.

The traditional way for measuring attention orienting is to calculate the difference between the mean RTs to detect targets at cued and uncued trials. However, examining the cueing effect in this way leads one to ignore another important influence on the cueing effect: the influence of previous trial types on current trial performance. Although some early studies suggested that very little visual information is explicitly retained across views (Grimes, 1996; Ballard, Hayhoe, \& Pelz, 1995), many recent studies have consistently showed that attention allocation is heavily influenced by the most recently viewed stimuli that were important for behavior (e.g., Chun \& Nakayama, 2000; Wolfe et al., 2003). For instance, Maljkovic and Nakayama (1994) found that in searching for a color singleton target, when target and nontarget colors are switched unpredictably from trial to trial, response in a trial is faster when the target color is the same as in the preceding trial than when it is different, a phenomenon that they called priming of pop-out (PoP). Besides color, this sequence effect on visual search performance has also been observed in investigations of other properties such as orientation (Hillstrom, 2000), shape (Lamy et al., 2006), location (Maljkovic \& Nakayama, 1996), and even emotional expression (Lamy, Amunts, \& Bar-Haim, 2008). PoP was generally believed to be afforded by implicit visual memory mechanisms without voluntary intervention (Kristjansson, 2006). Another good demonstration of sequence effects between trials is negative priming (e.g., Neill \& Valdes, 1992; Tipper, 2001), which refers to the phenomenon that a target stimulus is more slowly responded to on a current trial when the same stimulus was to be ignored on a previous trial. Negative priming was mainly explained as selective inhibition or episodic retrieval (Egner \& Hirsch, 2005). All of these studies showed that some crucial information from previous views could be used to guide attention allocation shortly afterward. Considering the important role of cue validity states (i.e., cue direction and target location is congruent or incongruent) in cueing paradigm, it is likely that after attention deployment to a target followed by a given cue, the relationship between the cue and the target (cued or uncued) in that trial can also be utilized, thus influencing subsequent cueing processes. Such sequence effects of cueing paradigm are important because they may reflect some temporal characteristics of attention orienting in humans and can provide better understanding of the cueing paradigm for future researches.
The first study to investigate the sequence effect of spatial cueing paradigm was reported by Maylor and Hockey (1987). They used a modified cueing paradigm with $500 \mathrm{~ms}$ SOA, in which the location cued by a peripheral cue (i.e., an exogenous cue) was maintained over 1,5 , or 30 trials during separate blocks. It was found that responses to a target at a given location were slowed when either the cue of the current trial or the target of the previous trial had been presented at that location. By using a standard exogenous cueing paradigm in which both the cue and the target occurred at random, Dodd and Pratt (2007) showed that the magnitude of IOR (i.e., RT inhibition effect) was greater when the target appeared at an uncued location on the previous trial, relative to when the target appeared at a cued location. This effect was due to the fact that participants were slower to respond to targets on cued trials when they were preceded by an uncued trial relative to a cued trial, and the participants were faster to respond to targets on uncued trials when they were preceded by an uncued trial relative to a cued trial. Dodd and Pratt interpreted the result as automatic memory check (Logan, 1988) in which information of previous trials was automatically retrieved from memory to facilitate performance on current trials. This explanation is in line with the implicit memory account for the phenomenon of PoP (Maljkovic \& Nakayama, 2000; Kristjansson, 2006) and the episodic memory retrieval account for negative priming (Egner \& Hirsch, 2005). While the results of Dodd and Pratt were obtained during the inhibition period of exogenous cueing (the SOA was $800 \mathrm{~ms}$ ), a recent study by Mordkoff, Halterman, and Chen (2008) extended the finding by showing that a similar sequence effect could be found at short $50 \mathrm{~ms} \mathrm{SOA}$ during the facilitation period of exogenous cueing: the cueing effect (i.e., RT facilitation effect) was reduced after an uncued than after a cued trial. This observation provided further evidence for the automaticity of the sequence effect, because the consensus in the literature has been that attention shifts on this timescale (i.e., less than $200 \mathrm{~ms}$ ) are not under any form of voluntary control (Klein, Kingstone, \& Pontefract, 1992, Nakayama \& Mackeben, 1989).

On the other hand, Jongen and Smulders (2006) investigated the sequence effect by a centrally presented arrow, a typical endogenous cue. In their experiment, the target appeared at cued location for $80 \%$ of all trials (i.e., a standard endogenous cueing task, which involved voluntary control of participants) and the cue-target SOA was $900 \mathrm{~ms}$. Similar to the findings from exogenous cueing tasks, it was found that the cueing effect was larger after a cued trial than after an uncued trial. However, since voluntary control was involved, Jongen and Smulders explained this sequence effect as momentary strategical adjustments, by which participants adapt their utilization of 
the cue depending on whether it is correctly or wrongly directed their attention on the previous trial. Specifically, a cued trial enhances the expectation for repetitions so that it is beneficial to direct attention to the cued location, whereas an uncued trial weakens this expectation or even promotes orienting to the uncued location. This explanation is completely different from the automatic memory check hypothesis that we mentioned earlier, even though a very similar phenomenon was interpreted. If both hypotheses are true, there should be two different mechanisms under the sequence effects of cueing paradigm, one for exogenous cues, which is reflexive, and one for endogenous cues, which is voluntary. However, due to the limitations of the task used in the study of Jongen and Smulders, they cannot rule out the possibility that the observed sequence effects are actually automatic and do not require voluntary control. Therefore, this study aimed to investigate whether the sequence effect of arrow cueing could emerge when voluntary control was not required to detect the target by using non-predictive arrow cues. If the sequence effect is based on strategy adjustments, the manner in which one adjusts when the cue is non-predictive should differ from the manner in which one adjusts when the cue is predictive. Specifically, Jongen and Smulders suggest that a cued trial would enhance the expectation for repetitions, whereas an uncued trial would weaken this expectation. With nonpredictive cues (i.e., the cue predicts $50 \%$ of the time), participants should either have no expectation or even have an expectation for alternations, because the more cued trials participants perceive the more uncued trials they should expect to subsequently appear. If the sequence effect is based on automatic memory check, the same sequence effect as previous studies will be expected.

There is another reason to believe that sequence effects by arrow cues do not require voluntary control, in addition to the evidence from the studies that used peripheral cues (Dodd \& Pratt, 2007; Mordkoff, Halterman, \& Chen, 2008). Though early studies suggested that endogenous cues, such as arrows, can shift attention only when they explicitly predicted the target location, many later studies have obtained cueing effects even with spatially non-predictive arrow cues (e.g, Hommel et al., 2001; Pratt \& Hommel, 2003; Ristic, Friesen, \& Kingstone, 2002; Tipples, 2002). Furthermore, several studies have found cueing effects at short SOAs when the arrow counter-predicts the target location (e.g., Hommel et al., 2001; Tipples, 2008), which means arrow cueing could not be suppressed in the same way as peripheral cueing. These results suggest that orienting in response to arrow cues is also reflexive and does not require voluntary control. Therefore, we expect that both the cueing effect within one trial and the sequence effect between trials could be triggered automatically by non-predictive arrow cues.
Another purpose of this study is to investigate the time course of sequence effects. From a memory perspective, there may be two major phases for the sequence processes: initial encoding phase in previous trials and later retrieval phase in current trials. In the former phase, the relationship between a cue and a target needs to be encoded into memory; in the later phase, the relationship information will be retrieved from memory to affect performance. Cue-target SOA is an important time factor in both phases. For peripheral cues, the sequence effect has been found at both short (Mordkoff, Halterman, \& Chen, 2008) and long SOAs (Dodd \& Pratt, 2007). For arrow cues, only a long SOA was investigated (Jongen \& Smulders, 2006).

One may expect sequence effects of arrow cues to also occur at short SOAs. However, this may not be the case. Previous studies have shown that the time course of arrow cueing is slower than that of peripheral cueing (e.g., Posner \& Cohen, 1984; Jonides, 1981; Frischen, Bayliss, \& Tipper, 2007), probably because symbolic cues like an arrow do not directly indicate a spatial location but rather require interpretation. Gibson and Bryant (2005) further showed that deliberate processing of the cue stimuli modulates orienting to uninformative central arrow cues. Thus, during the encoding phase, the relationship between an arrow and a target may not be encoded into memory if the perceiving time of the arrow is not sufficient. On the other hand, once the relationship information is encoded, it should be retrieved rapidly in an automatic way at both short and long SOAs depending on the automatic memory check hypothesis. Another possibility is that when the perceiving time of the arrow is short, the encoded relationship information from the previous trial will not be totally updated by the new relationship in the current trial, which in turn impairs the sequence effect in the next trial. Therefore, we expect that sequence effects of arrow cues were influenced by the SOAs of previous trials, but not influenced by the SOAs of current trials. Specifically, when the previous SOA is short, no sequence effect will be shown; however, when the previous SOA is long, sequence effects will be shown despite the length of the current SOAs.

In addition to the sequence effect of cue validity in arrow cueing, there was another finding about the influence of previous trials in the study of Jongen and Smulders (2006). They found that, following catch trials in which the target did not appear, the overall RTs were slowed compared with other trials, but the cueing effect was not influenced. They interpreted this result as a reduction in alertness and as support for the dissociation between spatial and temporal attentional mechanisms. In this study, we will examine whether the same result can be observed when relatively short SOAs are used and voluntary control is not required. 


\section{Method}

Participants

A total of 16 students (with a mean age of 26 years, range 21 to 29 years, 5 females) from Kochi University of Technology consented to participate in this experiment. All participants reported normal or corrected-to-normal vision and were naive as to the purpose of the experiment.

\section{Apparatus}

The stimuli were presented on a LCD display operating at a $60-\mathrm{Hz}$ frame rate and the display of the stimuli was controlled by E-Prime software (Schneider, Eschman, \& Zuccolotto, 2002). The participants were seated approximately $60 \mathrm{~cm}$ away from the screen.

\section{Stimuli}

A cross, subtending $1.5^{\circ}$, was placed at the center of the screen as a fixation point and remained at the screen during the whole experiment. The cue was an arrow to the left $(<<)$ or to the right $(>>)$ just around the central cross and was presented $1.5^{\circ}$ in height and $5^{\circ}$ in width. The target stimulus was a capital letter ' $\mathrm{T}$ ' measuring $1^{\circ}$ wide, $1^{\circ}$ high, and was presented $15^{\circ}$ away from the fixation point on the left or right side.

\section{Design}

The cue-target SOAs were 100 and $700 \mathrm{~ms}$. On each trial, cue direction, target location, and SOA duration were selected randomly and equally. There were five blocks with 100 trials each. In each block, 20 trials were catch trials in which the target did not appear. The participants were instructed not to respond if the target did not appear. Including 20 training trials, there were in total 520 trials for each participant. The RT of the first trial on each block was excluded from analysis because it was not preceded by any trials.

\section{Procedure}

Participants were instructed to keep fixating on the center of the screen. First, a fixation display appeared at the center of the screen for $2000 \mathrm{~ms}$, and then the cue stimulus appeared. After a certain cue-target SOA, a target letter ' $T$ ' appeared either at left or right until participants had responded or $1500 \mathrm{~ms}$ had elapsed. Participants were instructed to respond to the appearance of the target by pressing the 'SPACE' key as quickly and accurately as possible. Participants were also informed that the central stimuli did not predict the location in which target would appear and that they should try to ignore the central cues.

\section{Results}

Errors

The participants missed an average of about $0.1 \%$ of the targets and made false alarm errors on approximately $0.5 \%$ of the catch trials. Anticipations (RT of less than $100 \mathrm{~ms}$ ) and outliers (RT over $800 \mathrm{~ms}$ ) were classified as errors and were excluded from analysis. As a result, about $0.6 \%$ of all trials were removed. The error rates did not vary systematically and no signs of any speed-accuracy trade-off were observed.

\section{Cueing effects}

A two-way ANOVA with SOA (100 and $700 \mathrm{~ms}$ ), cue validity (cued and uncued) as within-participants factors was conducted on the RTs to investigate the overall cueing effects independent of previous trial types. There was a significant main effect of SOA, $F(1,15)=46.232$, $\mathrm{p}<.0001$, with RTs becoming shorter as the SOA was increased. The main effect of cue validity was also significant, $\mathrm{F}(1,15)=14.135, \mathrm{p}<.002$, indicating cueing effects, i.e., RTs were shorter at cued than at uncued trials. The interaction between SOA and cue validity was not significant $(\mathrm{p}>$.67). The average cueing effect (i.e., RTs of uncued trials - RTs of cued trials) was $8 \mathrm{~ms}$.

\section{Sequence effects of previous cue validity}

A three-way ANOVA with previous cue validity (pre-cued and pre-uncued), cue validity (cued and uncued), and cue direction (same or different than previous trial) as withinparticipants factors was conducted on the RTs to show the sequence effects and the influence of cue direction. There was a main effect of cue validity, $\mathrm{F}(1,15)=12.666$, $\mathrm{p}<.003$, indicating cueing effects. Importantly, there was a significant interaction between previous cue validity and cue validity, $\mathrm{F}(1,15)=10.489, \mathrm{p}<.006$, demonstrating that the cueing effect of current trials was significantly reduced following an uncued trial compared with a cued trial, i.e., a typical sequence effect reported by previous studies. Furthermore, neither the main effect of cue direction nor the previous cue validity $\times$ cue validity $\times$ cue direction interaction was significant, $\mathrm{F}(1,15)=1.573$, $\mathrm{p}>.22$, and $\mathrm{F}(1,15)=2.014, \mathrm{p}>.17$, respectively. No other factors or interactions were significant. A similar analysis was conducted to investigate the influence of target location (same or different than previous trial). Again, 
neither the main effect of target location nor the previous cue validity $\times$ cue validity $\times$ target location interaction was significant $(\mathrm{ps}>.15)$. In all, these results replicated sequence effects of cueing paradigm with non-predictive arrow cues and suggested that the sequence effects were not significantly influenced by the repetition/switch of cue direction and target location between trials.

Influence of previous and current SOAs on sequence effects

A four-way ANOVA was conducted on the RTs with previous SOA (100 and $700 \mathrm{~ms}$ ), previous cue validity (pre-cued and pre-uncued), current SOA (100 and $700 \mathrm{~ms}$ ), and cue validity (cued and uncued) as within-participants factors. Similar to the previous analysis, the cueing effect and the sequence effect were significant ( $\mathrm{ps}<.006$ ). The main effect for SOA was also significant, $F(1$, $15)=53.020, \mathrm{p}<.0001$, with RTs becoming shorter as the SOA was increased. Importantly, the previous SOA $\times$ previous cue validity $\times$ cue validity interaction was significant, $\mathrm{F}(1,15)=8.275, \mathrm{p}<.012$, indicating that the sequence effect was influenced by the previous SOAs. No other factors or interactions were significant. The RTs under different conditions were illustrated in Fig. 1.

To further investigate the influence of the previous SOAs on the sequence effect, a paired-samples t-test was used to compare the magnitude of cueing effects under different conditions. The magnitude of cueing effects are illustrated in Fig. 2. When the previous SOA was relatively short $(100 \mathrm{~ms})$, no significant sequence effects were observed for both current SOAs (both ps $>.79$ ). When the previous SOA was relatively long $(700 \mathrm{~ms})$, regular sequence effects were observed despite the length of current SOAs (both ps <.025). From the right part of Fig. 2 where sequence effects showed, we can see a tendency for sequence effects of current trials with a 700-ms SOA to be stronger than those with a $100-\mathrm{ms}$ SOA; however, this tendency was not significant $(\mathrm{p}>.37)$. The average sequence effect (i.e., cueing effects of pre-cued trialscueing effects of pre-uncued trials) was $19 \mathrm{~ms}$.

Influence of previous catch trials

As for the influence of preceding catch trials, a three-way ANOVA was conducted on the RTs with previous condition (the previous trial was a catch trial and it was not), SOA (100 and $700 \mathrm{~ms}$ ), and cue validity (cued and uncued) as within-participants factors. Similar to the previous analysis, the main effect of SOA was significant, $\mathrm{F}(1$, $15)=32.375, p<.0001$. Importantly, the main effect of previous condition was significant, $\mathrm{F}(1,15)=6.348$, $\mathrm{p}<.024$, indicating that after a catch trial, RTs were faster (393 ms) than after the average of the other trial types (407 ms). The main effect of cue validity was also significant, $\mathrm{F}(1,15)=8.864, \mathrm{p}<.009$, representing cueing effects. However, the interaction of previous condition and cue validity was not significant ( $\mathrm{p}>.60$ ). No other factors or interactions were significant. In sum, the results showed a facilitation effect of preceding catch trials on RTs and this effect was independent of the cueing effect.

\section{Control tasks}

Until now, we only investigated sequence effects from one previous trial. To investigate the influence of two trials prior to the current trial may be helpful to add more insight to the mechanisms under the sequence effects. The sequence effects could be divided into two groups depending on the repetition condition of previous two trials: repeated (e.g., both trial n-2 and trial n-1 is cued trials) or switched. No change in the magnitude of sequence effects between the two groups would be more consistent with the automatic memory check hypothesis, whereas a
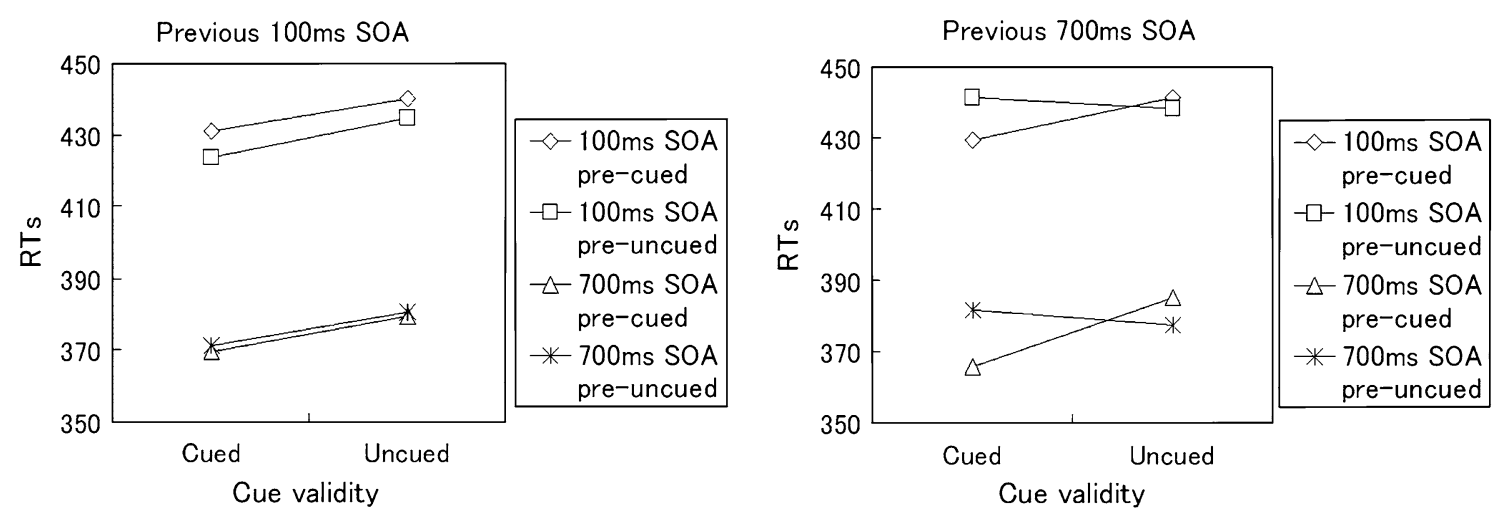

Fig. 1 Mean reaction times (RTs) under different previous and current cue validity, previous and current SOAs 
Fig. 2 The magnitude of cueing effects (RTuncuedRTcued) under different previous cue validity, previous and current SOAs. The asterisks mark the statistically significant differences (significant level 0.05). Error bars denote standard errors of the mean

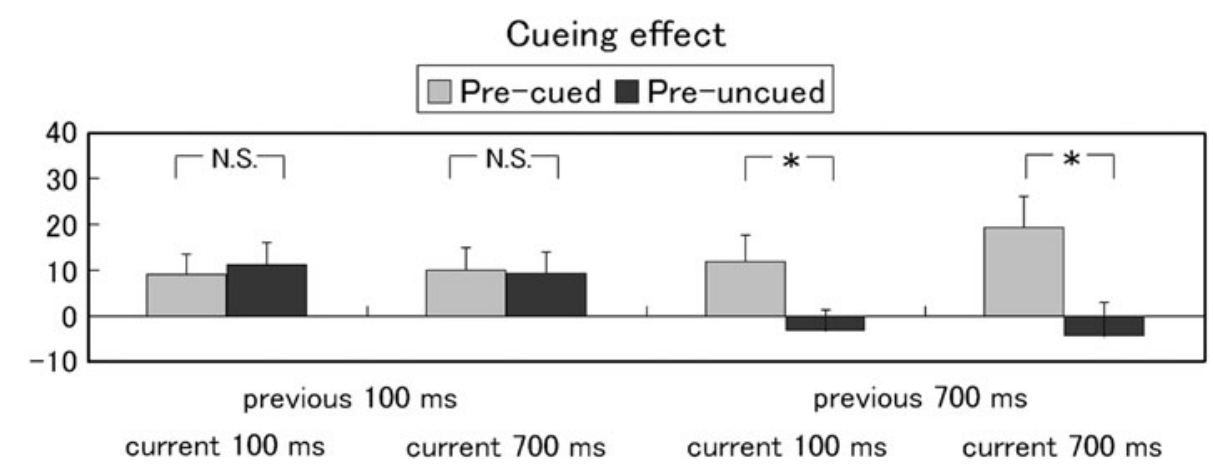

change in magnitude would be more consistent with the strategy adjustment hypothesis. However, an analysis based on current data is not reliable, because the preceding trials with a 100-ms SOA did not induce significant sequence effects and should be removed, which will result in a small sample size. Therefore, an additional control experiment was conducted by 12 participants. The SOA was $700 \mathrm{~ms}$ only and each participant completed a total of 273 trials (including 21 catch trials). A three-way ANOVA was conducted on the RTs with repetition condition between trial n-2 and trial n-1 (repeated and switched), cue validity of trial n-1 (pre-cued and pre-uncued), and cue validity of trial $\mathrm{n}$ (cued and uncued) as within-participants factors. The results showed a significant cueing effect and a significant sequence effect, $\mathrm{F}(1,11)=52.868, \mathrm{p}<.0001$, and $\mathrm{F}(1,11)=8.610, \mathrm{p}<.014$, respectively. Importantly, the influence of repetition condition on the sequence effect was not significant, $\mathrm{F}(1,11)=.05, \mathrm{p}>.82$, indicating that the sequence effect was not significantly influenced by the cue validity of trial $n-2$.

We did not observe additive sequence effects from the results of the previous control experiment, possibly because the memory to the cue validity was updated consecutively. However, we cannot rule out the possibility that sequence effects actually occurred on a response level, which abolished after each response. Therefore, in the second control experiment, a neutral-cue trial (instead of arrow, the cue is two vertical lines aside the fixation point without spatial meaning) was inserted into the middle of trial $\mathrm{n}-1$ and trial $\mathrm{n}$. If the sequence effect is indeed a phenomenon of memory, the sequence effect should still exist despite the additional responses in neutral-cue trials. Fourteen participants were tested and each of them completed a total of 200 trials (no catch trials were included). The cue-target SOA was $700 \mathrm{~ms}$. The results again showed a significant cueing effect and a significant sequence effect, $\mathrm{F}(1,13)=9.576, \quad \mathrm{p}<.009, \quad$ and $\mathrm{F}(1,13)=4.829$, $\mathrm{p}<.047$, respectively. These results suggest that the sequence effect observed in this study was indeed based on memory mechanisms.

\section{Discussion}

The present study investigated whether the sequence effect of cueing paradigm could be triggered by non-predictive arrow cues. The results showed that the sequence effect of arrow cueing could be observed when voluntary control was not required to detect the target (i.e., the arrow cue did not predict the target location). Additionally, when the previous SOA is short, no sequence effect was observed; however, when the previous SOA is long, the sequence effect was shown both at the short and long current SOAs. Furthermore, though both the study of Jongen and Smulders (2006) and the present study found that cueing effects were not influenced by a preceding catch trial, interestingly, we observed that following a catch trial, the overall RTs were facilitated, rather than slowed.

Sequence effects of cueing paradigm have been reported by several studies. Some of them (Dodd \& Pratt, 2007; Mordkoff, Halterman, \& Chen, 2008) have shown the sequence effect by using non-predictive peripheral cues. These results support the automatic memory check hypothesis (Logan, 1988), which suggests that when performing a task, participants are highly likely to automatically and unintentionally retrieve information from memory in order to facilitate current task performance. Specifically, when the previous trial type (cued or uncued) is consistent with the current trial type, performance will be facilitated, whereas when the previous and current trial types differ, performance is slowed due to the conflict between the two trial types. As a result, the magnitude of cueing effects (i.e., RT facilitation effect) was reduced during short SOAs and the magnitude of IOR was increased during long SOAs after an uncued trial compared with a cued trial. Similar phenomena of automatic memory mechanisms, such as priming of pop out (e.g., Lamy, Amunts, \& Bar-Haim, 2008; Maljkovic \& Nakayama, 2000; Kristjansson, 2006), and negative priming (e.g., Neill \& Valdes, 1992; Egner \& Hirsch, 2005), have also been reported by using other paradigms. All of these studies suggested that the sequential processes were afforded by 
implicit visual memory mechanisms, which operated in an automatic way without conscious intervention. A different hypothesis was proposed when arrow cues were tested by Jongen and Smulders (2006). They argued that the sequence effect was due to some strategies under the voluntary control of the participants. However, because the arrow cues predicted the target location in most of their experimental trials, their explanation may have confounded the voluntary cueing effect within one trial and the automatic sequence effect between trials. The present study extends the findings of Jongen and Smulders (2006) by demonstrating that sequence effects can be observed even when arrow cues are non-predictive to the actual target location. The strategy adjustment hypothesis will predict either no sequence effects or reversed sequence effects with non-predictive arrow cues. Therefore, the present results suggest that sequence effects of arrow cueing are not attributed to the voluntary control or explicit strategies of participants, but attributed to memory retrieval mechanisms, as suggested by the automatic memory check hypothesis.

Although the automatic memory check hypothesis may have revealed the nature of memory under the sequence effect, it does not explain the details of the sequential processes, such as what exactly happens within a spatial cueing task and how the information of previous trials is processed. Some recent studies by Hommel and his colleagues (Hommel, Proctor, \& Vu, 2004; Hommel, 2004) proposed a feature-integration account, which tried to explain the sequence effects in spatial attention tasks. The basic idea is that co-occurrence of a cue and a target leads to a transient representation of the relation in which their features (at least the features related to task) are spontaneously integrated without need for voluntary control. This relation would be reactivated in the next trial, and good performance would be expected if the same relation is repeated but interference would occur if it were alternated. According to this feature-integration account, the spatial meaning of the arrow cues and the spatial location of the targets in the present experiment were integrated to form a relation (either cued or uncued). This relation was retrieved in the next trial, and faster response was conducted when the same relation is repeated than when it is alternated.

One thing we need to point out is that the magnitude of the sequence effect observed in present study $(19 \mathrm{~ms})$ is very close to the results of previous studies (15 ms at Dodd and Pratt (2007); $17 \mathrm{~ms}$ at Mordkoff et al. (2008); around $20 \mathrm{~ms}$ at Jongen and Smulders (2006), perceived from their Fig. 4). The stable magnitude of sequence effects across very different experiments provided further evidence to support the automatic memory check hypothesis. In addition, considering the weak average cueing effect in the present study (only $8 \mathrm{~ms}$ ), it is not difficult to explain why the cueing effect of trials was completely lost when the previous trial was uncued with a $700 \mathrm{~ms}$ SOA. The answer is probably that the cueing effect was overpowered by the sequence effect.

In addition, we investigated the influence of previous SOAs and current SOAs on the sequence effect. It was found that when the previous SOA was short, no sequence effect was observed; but when the previous SOA was long, sequence effects were shown at both short and long current SOAs. This is a novel finding in the investigation of sequence effects of cueing paradigm. As mentioned in the introduction, the result can be explained by the different time course of two phases (i.e., initial encoding phase in previous trials and later retrieval phase in current trials) in the sequential processes. However, there are still some issues that need to be considered. First, the impairment of sequence effects when previous trials had a short SOA apparently contradicts the results of Mordkoff et al. (2008), in which the SOA was also very short but resembling sequence effects were observed. One critical difference between the two studies is the different attentional cues. The arrow cue involved in present study is perceptually different but spatially similar whereas the peripheral cue in their study is perceptual identical but the spatial location differs. Therefore, it is easy to integrate a peripheral cue with a target directly based on their spatial locations. On the contrary, arrow cues need to be discriminated before the spatial meaning of them can be acquired. It is widely accepted that though both peripheral cueing and arrow cueing can orient attention reflexively, their relative time courses are very different. In the same way, it is possible that though both peripheral cues and arrow cues could induce sequence effects automatically, some different processes have been involved, like different processing routes and different information that are encoded. This assumption is to some extent supported by the results of several pilot experiments, which are in preparation for a new research in our laboratory. The results showed that alternation of cue types (peripheral onset vs. central arrow) abolished overall sequence effects, whereas alternation of cue types (central gaze vs. central arrow) did not.

Second, we suggest that the influence of previous SOA may reflect a difficulty in encoding the relation between an arrow and a target with a short SOA relative to a long SOA. One may argue that the time interval between trials when a fixation point was presented for a full $2000 \mathrm{~ms}$ should be sufficient to let the relation be encoded. However, this view ignores the important fact that the cue and the target have disappeared before the $2000 \mathrm{~ms}$ inter-trial interval. Automatic processing is usually transient and stimulus-driven, so it is unlikely that the automatic encoding of the trial could occur without stimulus inputs during the inter-trial interval. 
Third issue is whether the influence of previous SOAs can be explained by the strategy adjustment hypothesis. In our opinion, the answer is probably no. Although similar explanation can be made, i.e., that a short perceiving time of the arrow may not be sufficient to enable participants to perceive the trial types on an initial trial, this notion faces the same question as why the perceiving cannot be done during a full 2000-ms inter-trial interval. This period of time should be enough for participants to discriminate between cued and uncued trials voluntarily. Another explanation could be that participants formed the expectation on an initial trial based on not only trial types, but also cue-target SOAs of that trial. Consequently, the participants adapted their utilization of the cue depending on if it correctly or wrongly directed their attention to a location on the previous trial, only when the cue-target interval of the previous trial was long enough. However, it is hard to believe that such a complex and resource-consuming strategy was maintained by participants across the whole experiment in spite of the fact that they explicitly knew the arrow cue was uninformative and SOAs were chosen randomly. In addition, the strategy explanation mentioned above will face many new questions. For example, how participants perceive the length of cue-target SOA as long or as short; is there a certain threshold or is it a relative adjustment? Therefore, at this stage, the influence of previous trial SOA cannot be used to discriminate between automatic and strategy hypotheses, we would like to consider this effect as originating from the different spatial representations between peripheral and central symbolic cues. On the other hand, the automatic memory check hypothesis is supported by the other results of present study, such as significant sequence effects by non-predictive arrow cues and the stable magnitude of the sequence effects across different studies. In all, though more systematic investigations are needed to reveal the precise mechanisms under the present results, our results are more consistent with the automatic memory check hypothesis and might reflect some different temporal characteristics of sequential memory mechanisms between peripheral cues and arrow cues.

Another effect that was examined in this experiment was the influence of preceding catch trials. Consistent with the findings of Jongen and Smulders (2006), we found that though the overall RTs were influenced following a catch trial, it did not influence the cueing effect. This observation supports the distinction between orienting and alerting processes of attention (e.g., Fernandez-Duque \& Posner, 1997; Posner \& Petersen, 1990). However, contrary to the findings of the present study, Jongen and Smulders found that overall RTs were delayed, rather than facilitated, after a catch trial. Besides their study, the overall delay in RTs after a catch trial has been reported by several other studies
(Alegria, 1978; Correa, Lupianez, \& Tudela, 2004), and it was attributed to a decrease in preparation for the target. The preparation refers to the general readiness to respond to an anticipated target stimulus after the occurrence of a warning cue. Therefore, if catch trials were considered as trials with extended cue-target SOA, a previous catch trial will reduce the target expectation of participants, resulting in a delayed RT at other SOAs.

Depending on the preparation account, arrow cues need to be utilized under some degree of strategy control to form expectancies about the target appearance. Therefore, it is not surprising to find that the RT delay effect of catch trials was not shown in the present experiment when voluntary control was not required and participants were encouraged to ignore the central cues. Another difference between the experiment of Jongen and Smulders and ours is the cuetarget SOA; while the single SOA of their experiment was relatively long, the present experiment used two SOAs with relatively short lengths. This setting may have increased the temporal uncertainty of the target appearance, which in turn reduced the influence of the attention preparation effect. In an exogenous cueing study, Los (2004) reported that target detection was slower when the cue-target SOA of the preceding trial was longer than the SOA of the current trial. However, at the shortest SOA $(100 \mathrm{~ms})$ of the two experiments that he conducted, he observed that responses after a preceding catch trial were faster, rather than slower, than that after a preceding long SOA. This observation is very similar to the finding of the present experiment. In all, these results suggest that a catch trial cannot be simply considered as a trial that extended cuetarget interval, and it may have a complex influence on the RTs depending on experimental contexts. Further investigation is needed to reveal the precise mechanisms under the RT effect of preceding catch trials.

The present study also has some implications on current and future investigations that involved cueing paradigm. As mentioned previously, a traditional way for measuring attention orienting is to calculate the difference between the mean RTs to detect targets at cued and uncued trials. This manipulation ignored the potential influence of trialby-trial effects. Though most cueing experiments included an equal number of cued and uncued trials, some researchers used a different proportion of cued trials relative to uncued trials in their experiments to investigate the influence of voluntary control on attention orienting (e.g., Driver et al., 1999; Friesen, Ristic, \& Kingstone, 2004). Sequence effects may have influenced their results. For example, when the cue predicts the target location with a rate of 80 percent, there will be more pre-cued trials than pre-uncued trials. As a result, larger average cueing effects for predictive cues than for non-predictive or counter-predictive cues are due in part to sequence effects, not only 
due to the voluntary control of participants. It is clearly important for future studies to take the influence of sequence effects into account when results are evaluated.

Finally, though both previous studies and our study focused on the sequence effect by traditional cues, such as peripheral cues or arrow cues, the sequential processing is not necessarily limited to these cue types. In another study (Qian, Shinomori, \& Song, 2011, in submission), we found significant sequence effects when a face stimulus looking left or right was used as a central cue. Another person's gaze has been considered as a special attentional cue for its biological significance (e.g., Friesen \& Kingstone, 1998, 2003). The findings that sequence effects could occur among very different attentional cues may suggest that sequential processing is a common phenomenon in daily life and the investigation into it will provide better understanding of human cognition systems.

In summary, the present experiment mainly demonstrated that sequence effects of cueing paradigm could be observed for non-predictive arrow cues. In addition, the sequence effects are influenced by the SOA of previous trials. Although the precise mechanisms under the different influence of previous SOAs between peripheral cues and arrow cues need further investigations, overall, our results support the automatic memory check hypothesis for the sequence effects of cueing paradigm more than the strategy adjustment hypothesis.

Acknowledgments The authors thank Michael Sharpe for English proofreading and thank Dr. Dodd and one anonymous reviewer for many helpful comments on a previous version of this manuscript. This research is supported by JSPS KAKENHI 20300081.

Open Access This article is distributed under the terms of the Creative Commons Attribution Noncommercial License which permits any noncommercial use, distribution, and reproduction in any medium, provided the original author(s) and source are credited.

\section{References}

Alegria, J. (1978). Sequential effects of catch-trials on choice reaction time. Acta Psychologica, 42, 1-6.

Ballard, D., Hayhoe, M., \& Pelz, J. (1995). Memory representation in natural tasks. Journal of Cognitive Neuroscience, 7, 66-80.

Chun, M., \& Nakayama, K. (2000). On the functional role of implicit visual memory for the adaptive deployment of attention across scenes. Visual Cognition, 7, 65-81.

Correa, A., j Lupianez, M. B., \& Tudela, P. (2004). Endogenous temporal orienting of attention in detection and discrimination tasks. Perception \& Psychophysics, 66, 264-278.

Dodd, M., \& Pratt, J. (2007). The effect of previous trial type on inhibition of return. Psychological Research, 71, 411-417.

Driver, J., Davis, G., Ricciardelli, P., Kidd, P., Maxwell, E., \& BaronCohen, S. (1999). Gaze perception triggers reflexive visuospatial orienting. Visual Cognition, 6, 509-540.
Egner, T., \& Hirsch, J. (2005). Where memory meets attention: Neural substrates of negative priming. Journal of Cognitive Neuroscience, 17, 1774-1784.

Fernandez-Duque, D., \& Posner, M. (1997). Relating the mechanisms of orienting and alerting. Neuropsychologia, 35, 477-486.

Friesen, C., \& Kingstone, A. (1998). The eyes have it! Reflexive orienting is triggered by nonpredictive gaze. Psychonomic Bulletin \& Review, 5, 490-495.

Friesen, C., \& Kingstone, A. (2003). Abrupt onsets and gaze direction cues trigger independent reflexive attentional effects. Cognition, 87, B1-B10.

Friesen, C., Ristic, J., \& Kingstone, A. (2004). Attentional effects of counterpredictive gaze and arrow cues. Journal of Experimental Psychology: Human Perception and Performance, 30, 319-329.

Frischen, A., Bayliss, A., \& Tipper, S. (2007). Gaze cueing of attention: Visual attention, social cognition, and individual differences. Psychological Bulletin, 133, 694-724.

Gibson, B., \& Bryant, T. (2005). Variation in cue duration reveals top-down modulation of involuntary orienting to uninformative symbolic cues. Perception \& Psychophysics, 67, 749-758.

Grimes, J. (1996). On the failure to detect changes in scenes across saccades. In K. Akins (Ed.), Vancouver studies in cognitive science: Vol.5. perception (p. 89-110). New York: Oxford University Press.

Hillstrom, A. (2000). Repetition effects in visual search. Perception \& Psychophysics, 62, 800-817.

Hommel, B. (2004). Event files: feature binding in and across perception and action. Trends in Cognitive Sciences, 8, 494-500.

Hommel, B., Pratt, J., Colzato, L., \& Godijn, R. (2001). Symbolic control of visual attention. Psychological Science, 12, 360-365.

Hommel, B., Proctor, R., \& Vu, K. (2004). A feature-integration account of sequential effects in the simon task. Psychological Research, 68, 1-17.

Jongen, E., \& Smulders, F. (2006). Sequence effects in a spatial cueing task: Endogenous orienting is sensitive to orienting in the preceding trial. Psychological Research, 71, 516-523.

Jonides, J. (1981). Voluntary versus automatic control over the mind's eye's movement. In J. B. Long \& A. D. Baddeley (Eds.), Attention and performance IX (p. 187-203). Hillsdale, NJ: Erlbaum.

Klein, R., Kingstone, A., \& Pontefract, A. (1992). Orienting of visual attention. In $K$. Rayner (Ed.), Eye movements and visual cognition. (p. 46-65). New York: Springer.

Kristjansson, A. (2006). Rapid learning in attention shifts: A review. Visual Cognition, 13, 324-362.

Lamy, D., Amunts, L., \& Bar-Haim, Y. (2008). Emotional priming of pop-out in visual search. Emotion, 8(2), 151-161.

Lamy, D., Carmel, T., Egeth, H., \& Leber, A. (2006). Effects of search mode and inter-trial priming on singleton search. Perception \& Psychophysics, 68, 919-932.

Logan, G. (1988). Toward an instance theory of automatization. Psychological Review, 95, 492-527.

Los, S. (2004). Inhibition of return and nonspecific preparation: Separable inhibitory control mechanisms in space and time. Perception \& Psychophysics, 66, 119-130.

Maljkovic, V., \& Nakayama, K. (1994). Priming of pop-out: I role of features. Memory \& Cognition, 22, 657-672.

Maljkovic, V., \& Nakayama, K. (1996). Priming of pop-out: II role of position. Perception \& Psychophysics, 58, 977-991.

Maljkovic, V., \& Nakayama, K. (2000). Priming of pop-out: III A short-term implicit memory system beneficial for rapid target selection. Visual Cognition, 7, 571-595.

Maylor, E. (1985). Facilitation and inhibitory components of orienting in visual space. In M. Posner \& O. Marin (Eds.), Attention and performance xi (p. 189-203). Hillsdale, NJ: Erlbaum.

Maylor, E., \& Hockey, R. (1987). Effects of repetition on the facilitatory and inhibitory components of orienting in visual space. Neuropsychologia, 25, 41-54. 
Mordkoff, J., Halterman, R., \& Chen, P. (2008). Why does the effect of short-SOA exogenous cuing on simple RT depend on the number of display locations? Psychonomic Bulletin \& Review, $15,819-824$.

Nakayama, K., \& Mackeben, M. (1989). Sustained and transient components of visual attention. Vision Research, 29, 1631-1647.

Neill, W., \& Valdes, L. (1992). Persistence of negative priming: Steady state or decay? Journal of Experimental Psychology: Learning, Memory, and Cognition, 18, 565-576.

Posner, M. (1980). Orienting of attention. Quarterly Journal of Experimental Psychology, 32, 3-25.

Posner, M., \& Cohen, Y. (1984). Components of visual orienting. In H. Bouma \& D. G. Bouwhuis (Eds.), Attention and performance xvii: Control of visual processing (p. 531-556). Hillsdale, NJ: Erlbaum.

Posner, M., \& Petersen, S. (1990). The attention system of the human brain. Annual Review of Neuroscience, 13, 25-42.

Posner, M., Snyder, C., \& Davidson, B. (1980). Attention and the detection of signals. Journal of Experimental Psychology: General, 109, 160-174.

Pratt, J., \& Hommel, B. (2003). Symbolic control of visual attention: The role of working memory and attentional control settings. Journal of Experimental Psychology: Human Perception and Performance, 29, 835-845.
Qian, Q., Shinomori, K., \& Song, M. (2011). Inter-trial effect of gaze cueing. (In submission).

Remington, R., Johnston, J., \& Yantis, S. (1992). Involuntary attentional capture by abrupt onsets. Perception \& Psychophysics, 51, 279-290.

Ristic, J., Friesen, C., \& Kingstone, A. (2002). Are eyes special? it depends on how you look at it. Psychonomic Bulletin \& Review, 9, 507-513.

Schneider, W., Eschman, A., \& Zuccolotto, A. (2002). E-prime user's guide. Pittsburgh, PA: Psychology Software Tools.

Tipper, S. (2001). Does negative priming reflect inhibitory mechanisms? A review and integration of conflicting views. Quarterly Journal of Experimental Psychology, 54A, 321-343.

Tipples, J. (2002). Eye gaze is not unique: Automatic orienting in response to uninformative arrows. Psychonomic Bulletin \& Review, 9, 314-318.

Tipples, J. (2008). Orienting to counterpredictive gaze and arrow cues. Perception \& Psychophysics, 70, 77-87.

Wolfe, J., Butcher, S., Lee, C., \& Hyle, M. (2003). Changing your mind: On the contributions of top-down and bottom-up guidance in visual search for feature singletons. Journal of Experimental Psychology: Human Perception and Performance, 29, 483-502. 